\title{
Electrical Conduction Mechanisms and Dielectric Constants of Nanostructure Zinc Indium Selenide Thin Films
}

\author{
Zeyada $\mathrm{HM}^{*}{ }^{1}$, Youssif $\mathrm{MI}^{1}$, El-Ghamaz NA ${ }^{1}$, and Aburoja MS ${ }^{2}$ \\ ${ }^{1}$ Department of physics, faculty of science at New Damietta, 34517, university of Damietta. Egypt \\ ${ }^{2}$ Department of physics, Faculty of science, Al-Jabl Al-Gharbi University, Libya
}

Received: 08 August 2015 / Accepted: 17 August 2015

* Corresponding author: (email: hzeyada@gmail.com)

\begin{abstract}
$\mathrm{ZnIn}_{2} \mathrm{Se}_{4}$ has polycrystalline structure in as synthesized powder form; it becomes nanocrystallites upon thermal deposition. The crystallite size increases by increasing annealing temperature. The radial distribution function showed that in the first short range order shell; Se atom is tetrahedral surrounded by a vacancy and three cationic sites occupied by the metal atoms in the ratio 2/3 of In and $1 / 3$ of $\mathrm{Zn}$. The medium range order region is attributed to In-In pairs having a layered structure of connected distorted octahedral. The direct current density-voltage characteristics for $\mathrm{Au} / \mathrm{ZnIn}_{2} \mathrm{Se}_{4} / \mathrm{Au}$ of planar structure revealed three conduction mechanisms depending on applied potential; namely they are consequently generation- recombination, Ohmic and exponential trap space charge limited conduction mechanisms. The AC electrical conductivity and dielectric relaxation of $\mathrm{ZnIn}_{2} \mathrm{Se}_{4}$ thin films in the temperature range 305-493 K and in frequency range $1 \mathrm{kHz}-4 \mathrm{M} \mathrm{Hz}$ has also been studied. Analysis of $\ln \sigma_{\mathrm{ac}^{-}} \ln \omega$ curves showed that band-type conduction process occurs in frequency range $<1945 \mathrm{~Hz}$. For frequencies $>1945 \mathrm{~Hz}, \sigma_{\mathrm{ac}}$ increases linearly with the increase in frequency and this is associated with hopping type conduction mechanism. Analysis of these results proved that conduction occurs by phonon assisted hopping between localized states and it is performed by bipolaron hopping mechanism. The temperature and frequency dependence of both the real and imaginary parts of the dielectric constant have been investigated..
\end{abstract}

Keywords: $\mathrm{ZnIn}_{2} \mathrm{Se}_{4}$ thin film; Conduction mechanisms; Dielectric constants

\section{Introduction}

$\mathrm{Znln}_{2} \mathrm{se}_{4}$ belongs to $A^{I I} B_{2}^{I I I} X_{4}^{V I}$ chalcopyrite family which has non-linear optical properties and technological applications such as light emitting diodes, laser screens, transistors and photovoltaic devices (Kale and Lokhande, 2005, and Pawar et al., 2011).

$\mathrm{ZnIn}_{2} \mathrm{Se}_{4}$ has potential applications in photo electronics, photovoltaics (Luengo et al., 1996 and Grilli et al., 1976), buffer layer in the fabrication of heterojunction solar cells (Babu et $a l ., 2011)$ and also in the development of electro- 
optical memory devices (Hendia and Soliman 1995, Filipowicz et al., 1981 and Edwards Soliman et al., 1999). Thin films of $\mathrm{Znln}_{2} \mathrm{Se}_{4}$ were prepared by different techniques such as thermal evaporation (Zeyada, et al., 2009). Spray pyrolysis (Yada. et al., 2008), electron deposition (Sun, 2009) and chemical bath deposition (Choe et al., 2009) techniques.

El- Nahass, et al. (El-Nahass, 2014) investigated dielectric and impedance spectral characteristics of bulk $\mathrm{ZnIn}_{2} \mathrm{Se}_{4}$. Materials are provided as thin films in device fabrication. The parameters controlling the properties of thin films are structure (Hagenmuller, 1990), composition (Sedeek et al., 1994 and Senthile et al., 2002), film thickness (El-Nahass et al., 2002 and Soliman et al., 1998), faults probability (Rouxel, et al., 1994) and the presence of impurities (Wiegers, 1989).

Annealing, substrate temperature and irradiation by ionizing particles or electromagnetic radiation influence structural parameters. In this work we investigate the role played by annealing temperature on structure, electrical conduction mechanisms and dielectric constants of zinc indium selenide thin films.

\section{Material and Methods}

\section{Material preparation and experimental techniques}

Ingots of $\mathrm{ZnIn}_{2} \mathrm{Se}_{4}$ were prepared by fusion of stoichiometry quantities of the pure elements in vacuum-sealed silica tubes; which were left at $1323 \mathrm{~K}$ for $10 \mathrm{~h}$ and then cooled to room temperature over $48 \mathrm{~h}$. Thin films of different thickness as were prepared by thermal evaporation technique using a high vacuum coating unit (Edwards 306 A, England). The working vacuum was kept at $10^{-5} \mathrm{~Pa}$ during deposition. The powder of $\mathrm{ZnIn}_{2} \mathrm{Se}_{4}$ was evaporated into thin films using quartz crucible heated by tungsten filament on to clean glass substrates for structural DC and AC electrical measurements. The deposition of films was done on substrates kept at room temperature $(300 \mathrm{~K})$. The film thickness and rate of deposition were controlled and monitored during deposition by film thickness monitor (model,TM-350 MAXTEK, Inc., USA). The deposition rate was controlled at $2 \mathrm{~nm} / \mathrm{s}$. The thickness of deposited films was also checked by interferometer technique (Hamza et al., 2003) Annealing of thin films for structural studies was performed in vacuum under a pressure of $10^{-3} \mathrm{~Pa}$ and at a temperature of $433 \mathrm{~K}$ with a soaking time of 2 hrs. The phase analysis of $\mathrm{ZnIn}_{2} \mathrm{Se}_{4}$ films with thickness of $600 \mathrm{~nm}$ was performed by X-ray diffraction technique. Phillips X-ray diffraction system (model X'pert pro) equipped with $\mathrm{Cu}$ target was used. A filtered $\mathrm{Cu} \mathrm{K}_{\alpha}$ radiation $(\lambda=$ $\left.1.5418^{\circ} \mathrm{A}\right)$ was applied. The $\mathrm{X}$-ray tube voltage and current were $40 \mathrm{kV}$ and $30 \mathrm{~mA}$, respectively. The radial distribution function (RDF) data were collected by using the same diffractometer using the Bragg-Brentauo Para-focusing technique. Highly monochromatic Mo radiation (wavelength $\lambda=0.709 \AA$ ) was used. The step scan mode was applied in the $2 \theta-$ range $(4-135)^{\circ}$. The step size $(\Delta 2 \theta)=0.04^{\circ}$ and the counting time was $10 \mathrm{sec}$. for each reading. The corresponding accessible maximum scattering vector magnitude, $\mathrm{V}$, was $16.50 \AA^{-1}$. The air scattering was avoided by a suitable applied arrangement of XRD system. The receiving and divergence slits were properly chosen in both small and large $2 \theta$ - ranges, in order to improve the qualities of the collected data. JEOL JSM-6360 scanning electron microscope (SEM) is used to determine the crystallite shape and size of $\mathrm{ZnIn}_{2} \mathrm{Se}_{4}$ thin films. The applied voltage is $30 \mathrm{kV}$.

The DC electrical measurements were performed for $\mathrm{ZnIn}_{2} \mathrm{Se}_{4}$ thin films with thickness of $600 \mathrm{~nm}$ in planar configuration between two gold electrodes which act as Ohmic contacts. The measurements of electrical resistance were performed using Keithley electrometer model 5617B. For AC measurements, $\mathrm{ZnIn}_{2} \mathrm{Se}_{4}$ thin films with thickness of $600 \mathrm{~nm}$ and area of 1.23 $\mathrm{mm}^{2}$ were sandwiched between two $\mathrm{Au}$ electrodes as lower and upper electrodes. A programmable automatic LCR bridge (Hioki LCR Hi tester meter model 3552-50, Japan) is used for determination of $\mathrm{AC}$ measurements parameters. This LCR meter can measure the capacitance, $C$, the resistance, $R$, the dissipation factor, $\tan \delta$, (displayed as $D$ ) and the quality factor, $Q$, in both of parallel and series modes. All the investigated measurements are represented on the screen of the bridge by a resistance, $R$, connected in parallel with a capacitance, $C$. The real part of the dielectric constant was calculated from the equation: $\varepsilon_{r}=\frac{C d}{\varepsilon_{o} A}$, where $\varepsilon_{o}$ is the permittivity of free space, $d$ is the film thickness and $A$ is its area. The dielectric loss $\varepsilon_{i}$ was also calculated from the equation: $\varepsilon_{i}=\varepsilon_{r} \tan \delta$ and $\sigma_{\mathrm{ac}}=\omega \varepsilon_{o} \varepsilon_{i}$. The $\mathrm{AC}$ 
measurements were carried out through temperature range from 333 to $473 \mathrm{~K}$ and frequency range from $1 \mathrm{kHz}$ to $4 \mathrm{MHz}$.

\section{Results and discussion}

\section{Structure of $\mathrm{ZnIn}_{2} \mathrm{Se}_{4}$ film}

The EDAX spectrum of thermally evaporated $\mathrm{ZnIn}_{2} \mathrm{Se}_{4}$ film in pristine condition had been studied in a previous work (Zeyada et al., 2009), only peaks of $\mathrm{Zn}$, In and Se are observed .The evaluated elemental composition are: Zn-9.42 at.\%, In-37.71 at.\% and Se-52.87at.\%. These results indicate that the structure of $\mathrm{ZnIn}_{2} \mathrm{Se}_{4}$ film keeps its stoichiometry after thermal evaporation. The XRD pattern of $\mathrm{ZnIn}_{2} \mathrm{Se}_{4}$ in powder form and annealed film condition, $433 \mathrm{~K}$ with soaking time of 2 hours, is shown in Fig.1. Fig.1 (a) shows a polycrystalline structure of $\mathrm{ZnIn}_{2} \mathrm{Se}_{4}$ in powder form. The Miller indices (hkl) are indicated on each diffraction peak using ICDD Card No. 80-0424. For all samples a major peak is observed at about $27.17^{\circ}$ and its equivalent inter-planar spacing is of $3.279 \AA$ which corresponds to reflection from (112) plane of tetragonal crystal system; this indicates that the nano-crystallites are preferentially oriented with (112) planes parallel to the surface of substrate. The tetragonal cell is of space group $\mathrm{I} \overline{4}$ with space parameters $\mathrm{a}=5.705 \AA \mathrm{A}, \mathrm{c}=11.448 \AA$ (Gastaldi et al., 1987). Fig.2 illustrates SEM image of pristine $\mathrm{ZnIn}_{2} \mathrm{Se}_{4}$ film; nano-crystallite structure is obtained with average crystallite size of $45 \mathrm{~nm}$ and it is homogenously distributed all over the film. Fig.1 (b) shows the influence of annealing temperature of $433 \mathrm{~K}$ with a soaking time of 2 hrs on $\mathrm{x}$-ray diffraction pattern of $\mathrm{ZnIn}_{2} \mathrm{Se}_{4}$ film, nanocrystallites are formed in the film. The crystallite size of $\mathrm{ZnIn}_{2} \mathrm{Se}_{4}$ in powder form and annealed thin film conditions have been determined on (112) diffraction peak using the well known Debye- Scherrer's (Cullity, 1979) equation:

$$
\text { C. } S=\frac{k \lambda}{\beta \cos \theta}
$$

where $\beta$ is the full width at half maximum (FWHM) intensity of the diffraction peak for (112), $\theta$ is the diffraction angle, $\lambda$ is the $\mathrm{x}$-ray wavelength $(1.5418 \AA$ ) and $k$ is a constant whose value is 0.98 . The determined crystallite size is
410, 45 and $84 \mathrm{~nm}$ for powder, pristine and annealed films, respectively. The radial distribution function, RDF, versus atomic distance $\mathrm{R}$ is represented in Fig. 3 for the pristine film. Up to $6.0 \AA$ the structural atomic correlations are good represented.

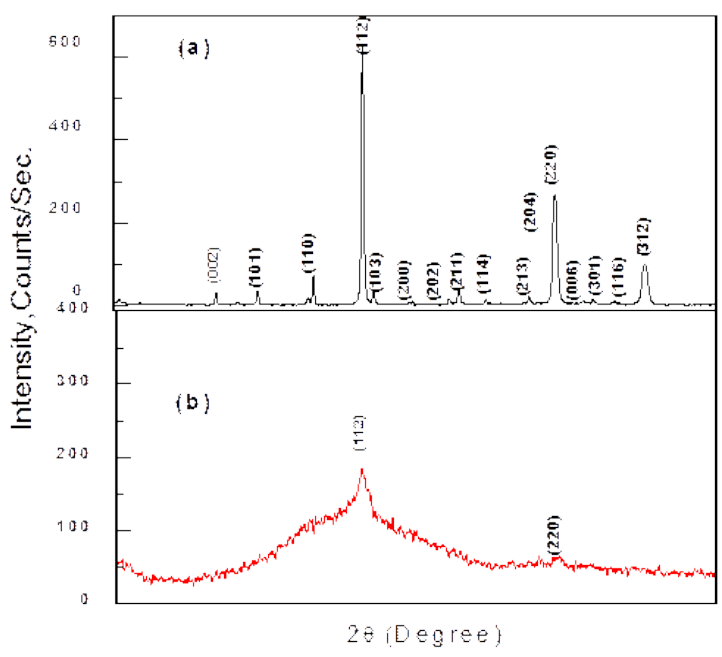

Fig. 1 XRD patterns for $\mathrm{ZnIn}_{2} \mathrm{Se}_{4}$ in: (a) powder form and(b) annealed thin film at $433 \mathrm{~K}$ with a soaking

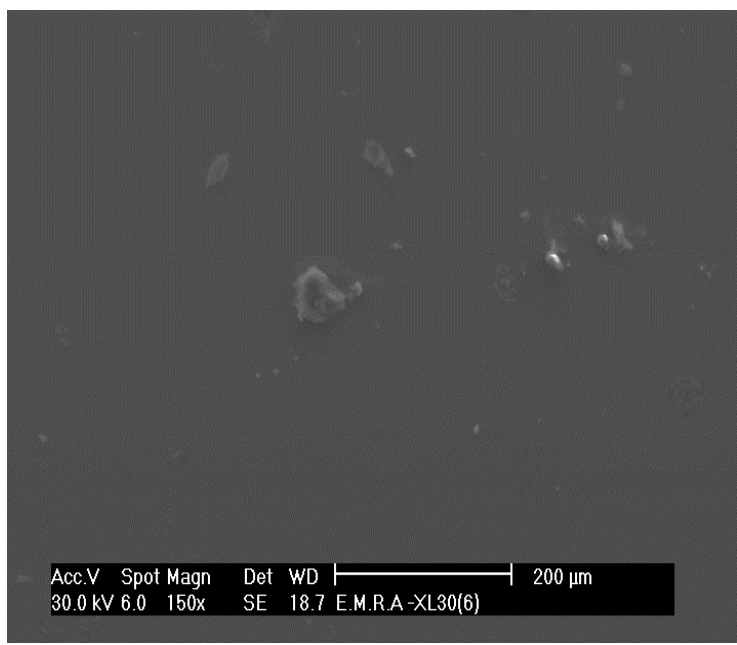

Fig. 2 Scanning electron microscope image for pristine $\mathrm{ZnIn}_{2} \mathrm{Se}_{4}$ thin film.

The first coordination shell for the sample is observed at almost $1.66 \AA$, this shell is stretched to be at $1.71 \AA$; in the first short range order ,SRO, shell Se atom is tetrahedral surrounded by a vacancy and three cationic sites occupied by the metal atoms in the ratio $2 / 3$ of In and 1/3 of Zn. The second peak is observed also for the film at around $2.60 \AA$ in form of a shoulder. This shoulder is due to In-Se pairs. The next peak is 
declared at $~ 3.20 \AA$ for the pristine film. This third coordination is also due to In-Se correlations inside the SRO. These arranged InSe pairs are in form of distorted tetrahedral. The next peak appears at $4.21 \AA$. This peak is in the medium range order, MRO, region and is attributed to In-In pairs having a layered structure of connected distorted octahedral. The RDF structural parameters for both SRO and MRO are collected in Table 1 . Concerning the bond angle distributions in SRO and MRO correlated pairs; the sample has a large value of $\varphi(\mathrm{Se}-\otimes-\mathrm{Se})$ of $103.86^{\circ}$. The bond angle of (In-Ŝe-In) correlations is $\sim 75^{\circ}$ for the pristine film.

\section{DC electrical measurements}

The DC electrical conductivity, $\sigma_{\mathrm{dc}}$, for planar samples of $\mathrm{ZnIn}_{2} \mathrm{Se}_{4}$ has been related to the temperature by the following equation (Mott, 1987)

$$
\sigma=\sigma_{0} \exp \left[\frac{E_{g}}{2 K_{B} T}\right]
$$

where $\sigma_{\mathrm{o}}$ is the pre-exponential factor, $\mathrm{E}_{\mathrm{g}} / 2$ is the thermal activation energy for this process, $\mathrm{T}$ is the temperature expressed in Kelvin and $K_{B}$ is the Boltzmann constant. The plot of $\ln \left(\sigma_{\mathrm{dc}}\right)$ versus reciprocal of temperature $(1 / \mathrm{T})$ in the temperature range $357-448 \mathrm{~K}$ for planar samples of $\mathrm{ZnIn}_{2} \mathrm{Se}_{4}$ film thickness of $600 \mathrm{~nm}$ is shown in Fig. 4. A linear relationship between in $\left(\sigma_{\mathrm{dc}}\right)$ and $1 / \mathrm{T}$ is observed; The activation energy, $\mathrm{E}_{\mathrm{g}} / 2$, is calculated from the slope of the straight line and found to be $0.96 \mathrm{eV}$, It is noted that the obtained value of thermal activation energy is half the value of energy gap of $\mathrm{ZnIn}_{2} \mathrm{Se}_{4}$ (Zeyada et al., 2009 and Choe, et al., 2009) $\sigma_{\mathrm{o}}$ is obtained from the intercept of the straight line with ordinate axis.

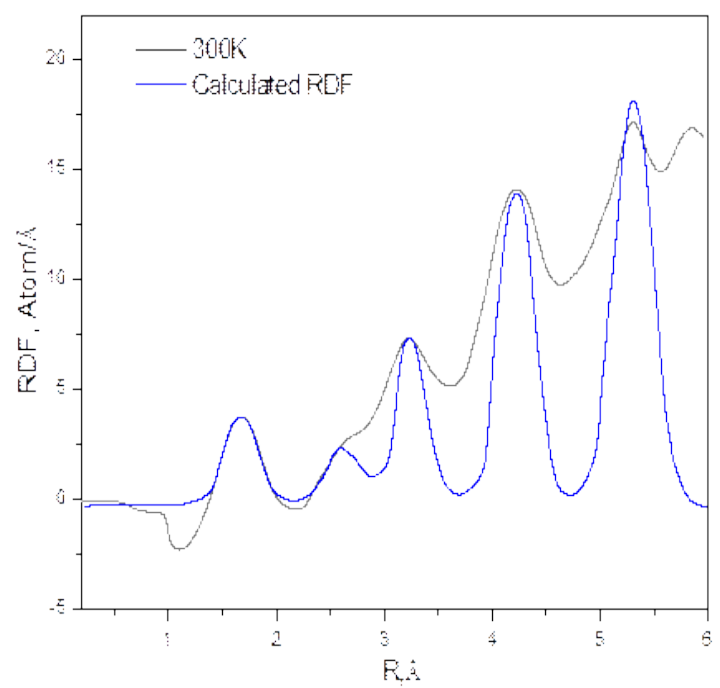

Fig. 3 RDF simulation of $\mathrm{Znln}_{2} \mathrm{Se}_{4}$ in the real space, the substrate temperature is $300 \mathrm{~K}$.

Table 1 Parameters of RDF for $\mathrm{ZnIn}_{2} \mathrm{Se}_{4}$ films deposited at substrate temperature of $300 \mathrm{~K}$ (film thickness $=300 \mathrm{~nm}$ )

\begin{tabular}{|l|l|l|l|l|l|l|l|l|l|c|}
\hline $\begin{array}{l}\text { Substrate } \\
\text { Temperature }\left(\mathrm{T}_{\mathrm{s}}\right)\end{array}$ & $\mathrm{R}_{1}, \AA$ & $\mathrm{N}_{1}$, atoms & $\begin{array}{c}\mathrm{R}_{2}, \AA \\
(\text { shoul. })\end{array}$ & $\begin{array}{c}\mathrm{N}_{2}, \\
\text { atoms }\end{array}$ & $\mathrm{R}_{3}, \AA$ & $\begin{array}{c}\mathrm{N}_{3}, \\
\text { atoms }\end{array}$ & $\mathrm{R}_{4}, \AA$ & $\mathrm{N}_{4}$,atoms & $\mathrm{R}_{5}, \AA$ & $\begin{array}{c}\mathrm{N}_{5}, \\
\text { atoms }\end{array}$ \\
\hline $298 \mathrm{~K}$ & 1.664 & 3.455 & 2.621 & ---- & 3.231 & 6.661 & 4.211 & 10.610 & 5.291 & 12.531 \\
\hline Pair type & $\begin{array}{l}\mathrm{Se}-\otimes \\
-\mathrm{Zn} \\
-\mathrm{In} \\
-\mathrm{In}\end{array}$ & & $\mathrm{In}-\mathrm{Se}$ & & $\mathrm{In}-\mathrm{Se}$ & & $\mathrm{In}-\mathrm{In}$ & & ------ & \\
& & & & & & & & & \\
\hline
\end{tabular}

$\otimes$-Vacancy; $\mathrm{R} \pm 0.03 \AA ̊$; \pm 0.20 atoms

Fig. 5 illustrates room temperature current density-voltage (J-V) characteristics for $\mathrm{Au} /$ $\mathrm{ZnIn}_{2} \mathrm{Se}_{4} / \mathrm{Au}$ of planar structure for samples of $\mathrm{ZnIn}_{2} \mathrm{Se}_{4}$.It is clear that there are different conduction mechanisms operating in the device depending on the applied voltage. The $\log \mathrm{J}-$ $\log \mathrm{V}$ relation is linear. There are three distinct voltage regions; in the low voltage region, the value of the slope is equal to 0.164 and this corresponds to the generation- recombination of current carriers. At potential bias $0.164<\mathrm{V}<$ 2.77, the dark $\log \mathrm{J}-\log \mathrm{V}$ relation is linear with slope equal to 0.96 , indicating that the conduction mechanism may be described by Ohm's law (Saleh et al., 1993 and Krishnakumar and Menon 1997) where the current density is given by: 


$$
\mathrm{J}=\mathrm{en}_{\mathrm{o}} \mu \frac{V}{d}
$$

where $\mathrm{e}$ is the electronic charge, $\mathrm{n}_{\mathrm{o}}$ is the carrier density, $\mu$ is the carrier mobility, $\mathrm{V}$ is the applied potential. The concentration of thermally generated electrons in the conduction band, $\mathrm{n}_{\mathrm{o}}$, can be calculated from the Ohmic region of the $\mathbf{J}$ - V characteristics for a $\mathrm{ZnIn}_{2} \mathrm{Se}_{4}$, taking into account that the $\mathrm{ZnIn}_{2} \mathrm{Se}_{4}$ is n-type semiconductor (Zeyada et al., 2009) the electron mobility is $8 \times 10^{-3} \mathrm{~cm}^{2} \quad \mathrm{~V}^{-1} \mathrm{~s}^{-1}$ at room temperature (Abdullayev et al., 1990) and film thickness is $600 \mathrm{~nm}$. The value of $\mathrm{n}_{\mathrm{o}}$ for $\mathrm{ZnIn}_{2} \mathrm{Se}_{4}$ thin films is calculated and found to be $4.5 \times 10^{18}$ $\mathrm{m}^{-3}$.

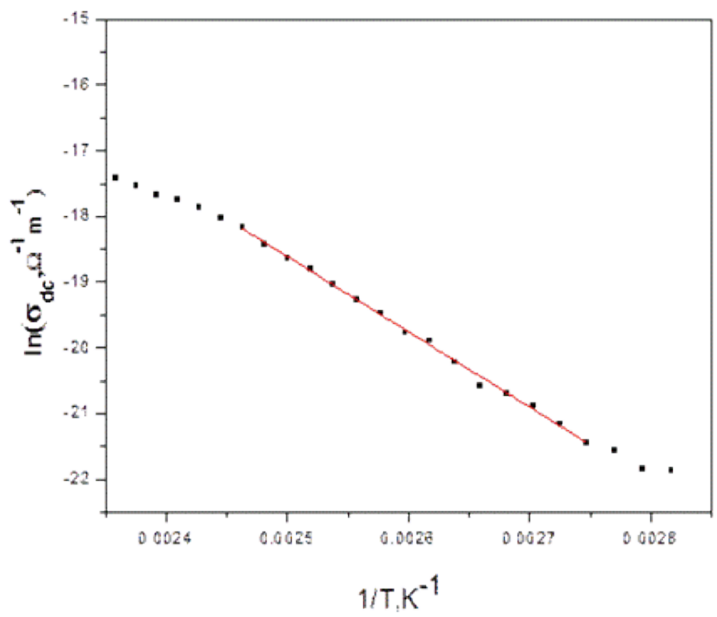

Fig. 4 A plot of $L n \sigma_{d c}$ versus $1 / \mathrm{T}$ for $\mathrm{Znln}_{2} \mathrm{Se}_{4}$ thin film.

Ohm's law breaks down at potential bias $>2.77 \mathrm{~V}$. above this potential, the slope of $\log \mathrm{J}-$ $\log \mathrm{V}$ curve is 4.55 , (Fig. 5) indicating that exponential trap space charge limited conduction (SCLC) is the operating conduction mechanism (Gao et al., 2001and Abdel Malik and AbdelLatif 1997 and Mazen et al., 1997) the current density is given by:

$$
\mathrm{J}_{\text {SCLC }}=\mathrm{e} \mu N_{c}\left(\frac{\varepsilon}{e H_{o} k_{\beta} T_{t}}\right)^{l} \frac{V^{l+1}}{d^{2 l+1}}
$$

where $\mathrm{N}_{\mathrm{c}}$ is the effective density of states at the conduction band, $H_{0}$ is the trap concentration per unit energy range at the conduction band edge, $\varepsilon$ is the permittivity of $\mathrm{ZnIn}_{2} \mathrm{Se}_{4}$, films, $(\ell+$ 1) represents the power law exponent and $\ell=T_{t}$ $/ T$; where $T_{t}$ is the temperature parameter characterizing the exponential trap distribution given by (Gao et al., 2001) as:

$$
\mathrm{H}(\mathrm{E})=\mathrm{H}_{\mathrm{o}} \exp \left(-\frac{E}{k_{B} T_{t}}\right),
$$

where $\mathrm{H}(\mathrm{E})$ is the concentration of traps per unit energy range as a function of energy, $E$, under the valence band edge. The total concentration of traps, $\mathrm{N}_{\mathrm{t}(\mathrm{e})}$, is given according to (Gao et al., 2001 and Abdel Malik and Abdel latif 1997) by:

$$
N_{t(e)}=H_{o} k_{B} T_{t}
$$

It is interesting to note that there is a transition voltage separating Ohmic conduction mechanism from SCLC mechanism. From Eq. (4) a slope of 4.55 implies that $\ell=3.55$ and the temperature parameter is $\mathrm{Tt}=1065 \mathrm{~K}$ for $\mathrm{T}=300 \mathrm{~K}$.

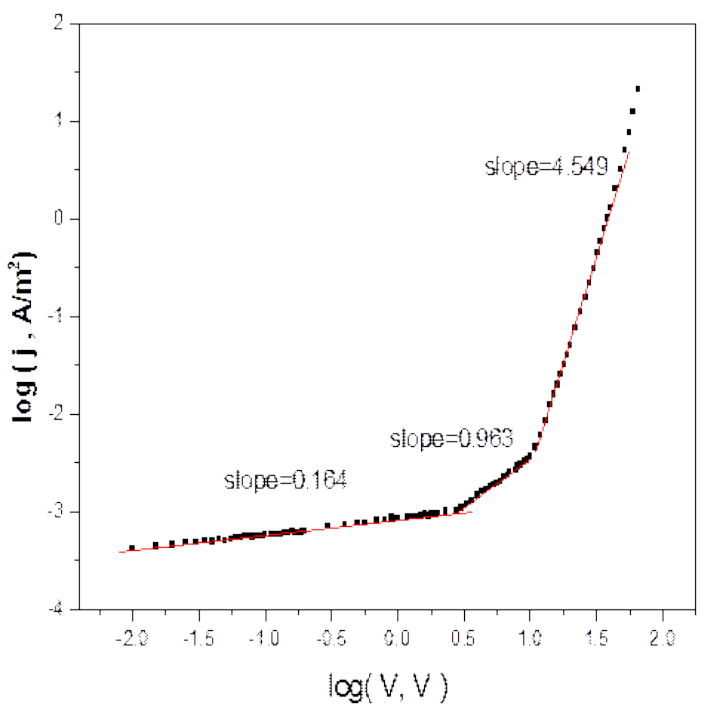

Fig. 5 The relation between $\log \mathrm{J}-\log \mathrm{V}$ for $\mathrm{Znln}_{2} \mathrm{Se}_{4}$ thin films.

\section{AC electrical measurements}

The AC electrical conductivity, $\sigma_{\mathrm{ac}}$, as a function of temperature and frequency has been investigated. The measurements were performed for $\mathrm{ZnIn}_{2} \mathrm{Se}_{4}$ films of thickness $600 \mathrm{~nm}$ sandwiched between two gold electrodes. The variation of $\ln \sigma_{\mathrm{ac}}$ versus reciprocal temperature for $\mathrm{ZnIn}_{2} \mathrm{Se}_{4}$ films in the temperature range 300$500 \mathrm{~K}$ and in the frequency range $1 \mathrm{kHz}-4 \mathrm{M} \mathrm{Hz}$ is depicted in Fig. 6; it shows a frequency and temperature dependence of AC conductivity. The $\mathrm{AC}$ conductivity increases with the increase of both temperature and frequency. There are two domains of conductivity depending on 
temperature. The activation energy for each domain is calculated according to:

$$
\sigma_{a c}=\sigma_{o o} \exp \frac{E_{a}}{k_{B} T} \text { and is depicted in Fig.7 }
$$

as a function of frequency. The activation energy, $E_{a}$, decreases with increasing frequency. The activation energy in high temperature region is greater than that in low temperature region. The obtained value of the AC activation energy at any frequency is lower than the DC activation energy over the same range of temperature. This is clear since the charge carriers in the DC conduction choose the easiest paths, which include some large jumps, while this is not so important in the AC conduction (Mazen, 1997) The increase of the applied field frequency enhances the electronic jumps between the localized states, consequently the activation energy decreases with increasing frequency. The small values of the AC activation energy compared with that of DC activation energy and the increase of $\sigma_{\mathrm{ac}}$ with frequency confirm that the hopping conduction is the dominant mechanism.

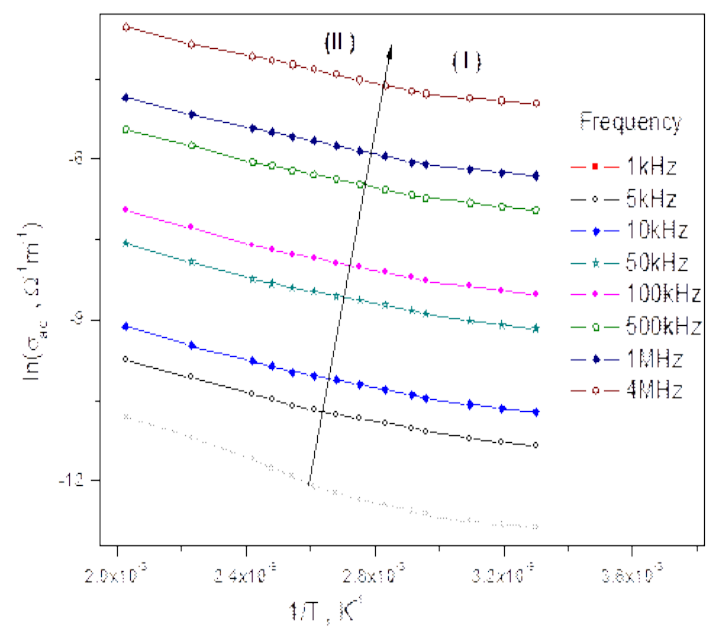

Fig. 6 A plot of $L n \sigma_{d c}$ versus $1 / T$ in the frequency range $1 \mathrm{kHz}-4 \mathrm{MHz}$ for $\mathrm{Znln}_{2} \mathrm{Se}_{4}$ thin film.

Fig. 8 illustrates the data of $\log \sigma_{\mathrm{ac}}$, versus $\log \omega$ for $\mathrm{ZnIn}_{2} \mathrm{Se}_{4}$ thin film of thickness $600 \mathrm{~nm}$ at different temperatures and sandwiched between two gold electrodes. As observed from the curves, AC conductivity increases with the rise in temperature and it decreases nonlinearly with increasing frequency in the frequency range from 308 to $1945 \mathrm{~Hz}$. For frequencies > $1945 \mathrm{~Hz}$, $\sigma_{\mathrm{ac}}$ increases linearly with the increase in frequency. Similar behavior has been observed in some semiconductor materials (Shafai and
Gould, 2007, and Vishnuvardhan et al., 2006). Generally, decrease of conductivity with increasing frequency is associated with band-type conduction process, while increasing conductivity with increasing frequency is associated with hopping type conduction mechanism (Azim-Araghi, 2007and Azim et al., 1996) . Therefore, for the range of frequencies < $1945 \mathrm{~Hz}$ the band theory can be applied and for the range of frequencies $>1945 \mathrm{~Hz}$, the hopping mechanism is suitable for $\mathrm{ZnIn}_{2} \mathrm{Se}_{4}$ thin films. This indicates that AC conductivity is governed by the power law:

$$
\sigma_{a c}=A \omega^{s},
$$

where $A$ is a constant and $s$ is called the frequency exponent. The values of frequency exponent were obtained for each curve from the slope of linear parts at high frequencies of the log $\sigma_{\mathrm{ac}}$ versus $\log \omega$ relation for different temperatures. A plot of frequency exponent versus temperature is shown in Fig.9, from which we can see that $\mathrm{s}$ decreases with the increase in temperature and it approaches unity as the temperature decreases to $300 \mathrm{~K}$. Such a behavior of $s$ with temperature indicates that correlated barrier hoping $(\mathrm{CBH})$ (Elliott, 1978) is the operating conduction mechanism in $\mathrm{ZnIn}_{2} \mathrm{Se}_{4}$ films. $\mathrm{CBH}$ model for $\mathrm{AC}$ conductions has been developed initially by Pike for single electron hopping This model is extended by Elliott for two electrons hopping (Elliott, 1987 and Ardona et al., 1986) for neighboring sites at a separation $R_{\omega}$, the Coulomb wells overlap, resulting in lowering of the effective barrier from its maximum height $W_{M}$ to a value $W$ given by

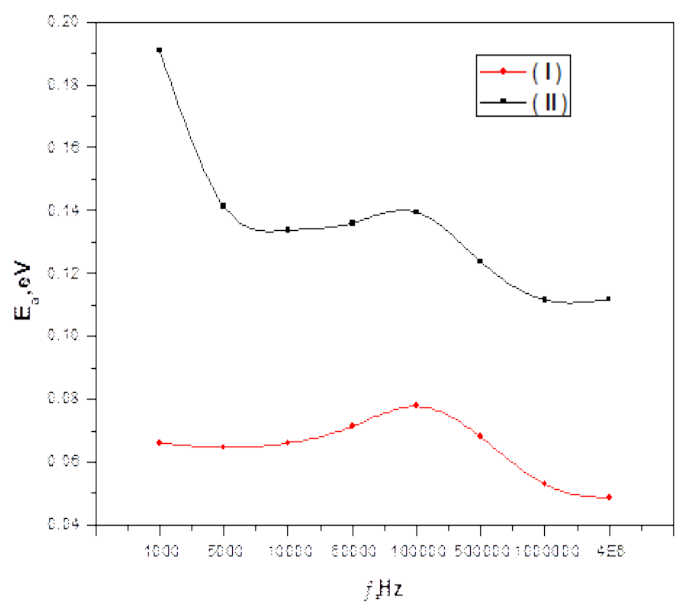

Fig. 7 Variation of activation energy, $\Delta \mathrm{E}_{\mathrm{ac}}$ versus frequency $f$, , for $\mathrm{Znln}_{2} \mathrm{Se}_{4}$ thin films; (I) low temperature region and (II) high temperature region. 


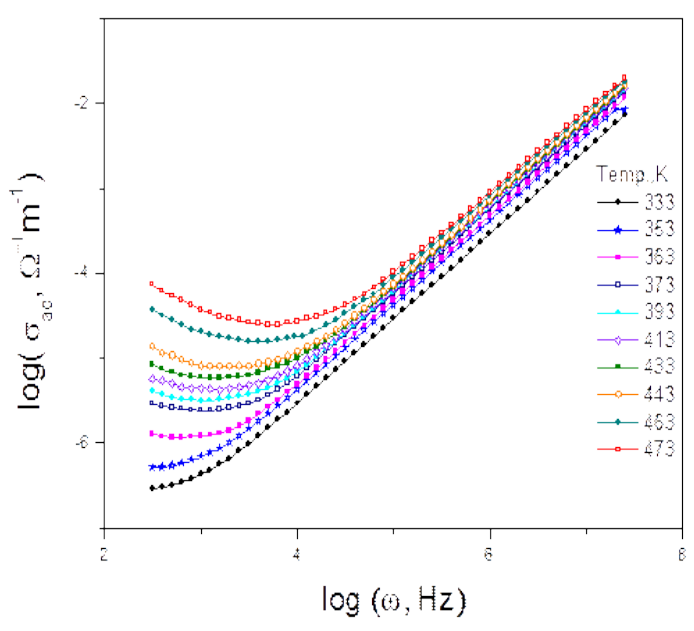

Fig. 8 The relation between $\log \sigma_{\mathrm{ac}}-\log \omega$ for $\mathrm{Znln}_{2} \mathrm{Se}_{4}$ thin films in the temperature range 333$473 \mathrm{~K}$.

$$
W=W_{M}-\frac{e^{2}}{\pi \varepsilon_{1} \varepsilon_{o} R_{\omega}}
$$

The equations defining $\sigma_{\mathrm{ac}}$ and $R_{\omega}$ are given in (Elliott, 1987) The first approximation for the frequency exponent $s$ according to this model of Elliott (Elliott, 1987) is given by:

$$
s=1-\frac{6 k_{B} T}{W_{m}}
$$

\section{Dielectric measurements}

Figure 10 shows the capacitance-frequencytemperature, C-f-T, characteristics of $\mathrm{ZnIn}_{2} \mathrm{Se}_{4}$ sandwich thin film with thickness of $600 \mathrm{~nm}$ in the frequency range $51 \mathrm{~Hz}-4.4 \mathrm{MHz}$ and at various constant temperatures ranging from 333 $473 \mathrm{~K}$. It can be seen that for a constant frequency as the temperature increases the capacitance increases as well and for a certain temperature it is also observed that the capacitance decreases nonlinearly with the increase in frequency up to a certain frequency, $f_{c}$, after which it arrives to a constant capacitance value. The capacitance is shown to be strongly frequency dependent at relatively low frequencies and high temperatures, but became less at high frequencies and at low temperatures. This result is associated with the release of charge carriers from relatively deep traps (Azim, 1996 and Gould et al., 1996) Such a behavior of the capacitance of $\mathrm{ZnIn}_{2} \mathrm{Se}_{4}$ thin film with frequency and temperature is the ordinary one for some of the semiconductor materials (Zeyada, 2011, and El-Nahass et al., 2005).

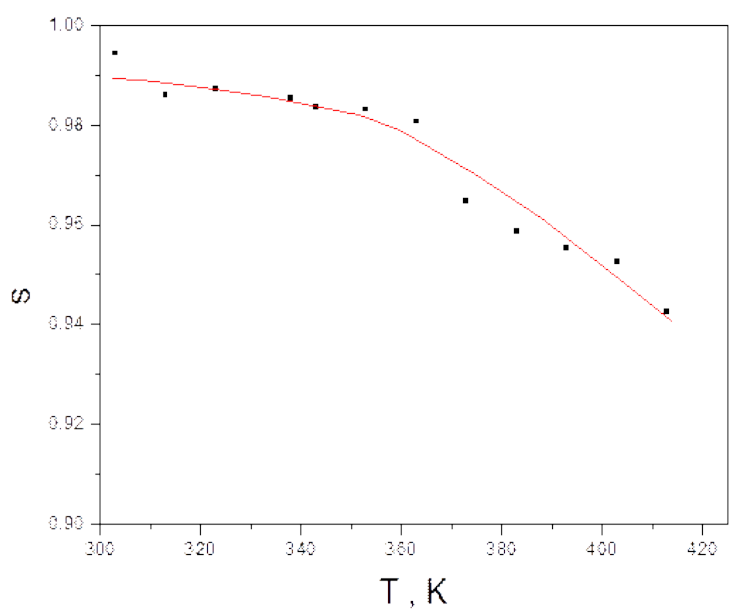

Fig. 9 Variation of exponent factor, $S$, versus temperature for $\mathrm{Znln}_{2} \mathrm{Se}_{4}$ thin films in the temperature

The temperature dependence on $s$ shown in Fig. 9 is consistent with Eq. 9 indicating that the dominance of $\mathrm{CBH}$ model as electrical transport mechanism for $\mathrm{ZnIn}_{2} \mathrm{Se}_{4}$ thin films.

Figs. 11 and 12, depict the dependence of the real, $\varepsilon_{\mathrm{r}}$, and the imaginary, $\varepsilon_{\mathrm{i}}$, parts of the dielectric constant on the frequency and temperature for $\mathrm{ZnIn}_{2} \mathrm{Se}_{4}$ film. The value of $\varepsilon_{\mathrm{r}}$ is greater than the value of $\varepsilon_{\mathrm{i}}$ at the same temperature and frequency. $\varepsilon_{\mathrm{r}}$ and $\varepsilon_{\mathrm{i}}$ have the same response to temperature and frequency where they increase with increasing temperature and decrease with the increase of frequency. The values of $\varepsilon_{\mathrm{r}}$ and $\varepsilon_{\mathrm{i}}$ is great at high temperatures as well as at low frequencies. The behavior of $\varepsilon_{\mathrm{r}}$ and $\varepsilon_{\mathrm{i}}$ at different temperatures can be explained by means of the dielectric polarization mechanism of the material. The temperature variation of the dielectric constant for the perfect solid dielectrics depends on three factors that are related to the thermal expansion and polarizability of the material. The first factor represents the decrease in the number of polarizable particles per unit volume as the temperature increases and has a direct effect on the volume expansion. The second one signifies the increase in the polarizability of a constant number of particles as the volume increases and the third factor denotes the change in polarizability due to temperature changes at a constant volume. At high frequency region, the variation in the field is very rapid for the dipoles to align themselves, so their 
contribution to the polarization and hence, to dielectric permittivity can become negligible. Therefore, the dielectric permittivity, $\varepsilon_{\mathrm{r}}$, decreases with increasing frequency. The decrease of the dielectric constant, $\varepsilon_{\mathrm{r}}$, can also be explained from interfacial polarization.

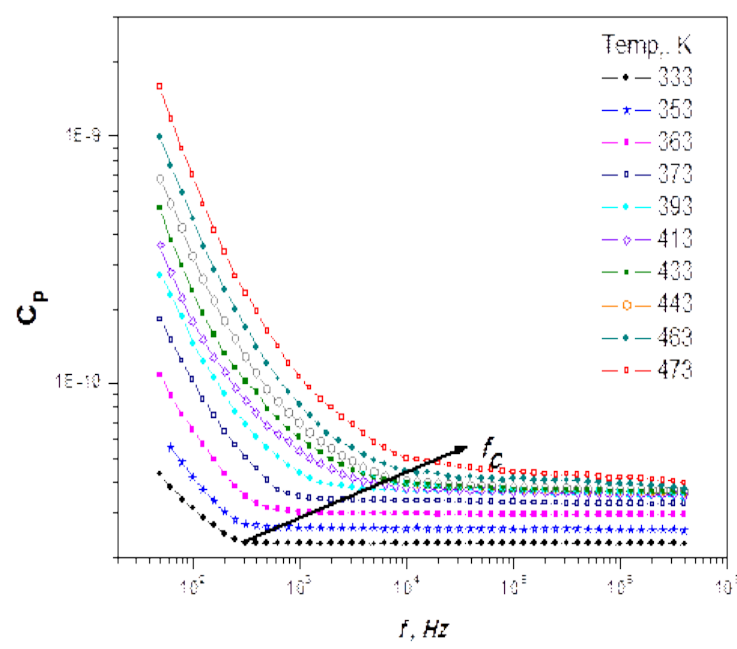

Fig. 10 Variation of parallel capacitance, $C_{p}$, with frequency $f$, for $\mathrm{Znln}_{2} \mathrm{Se}_{4}$ thin films in the temperature range $333-473 \mathrm{~K}$.

The interfacial polarization arises as a result of difference in conducting phase, but is interrupted at grain boundary due to lower conductivity. Generally in polycrystalline materials, the grains exhibits semi conducting behavior while the grain boundary are insulators. The imaginary part, $\varepsilon_{\mathrm{i}}$, of the dielectric constant corresponds to a current density within the dielectric that is no longer exactly $\pi / 2$ out of phase with the electric field. It is responsible for the dissipation in the dielectric at the specific frequencies as depicted in Fig.12. It is known that the contribution to the dielectric loss generally consists of a conduction and relaxation components. The higher values of $\varepsilon_{\mathrm{i}}$ at relatively low frequency may be attributed to the contribution arising from both the conduction and relaxation losses. At higher frequencies the relaxation losses are the only sources of dielectric loss. It is also noticed that $\varepsilon_{\mathrm{i}}$ increases with the increase in temperature. As the temperature increases, the relaxation loss component reduces and the conduction loss component increases more rapidly. $\varepsilon_{\mathrm{i}}$ is directly proportional to $\sigma_{a c}$ at constant frequency as the temperature increases the electrical conductivity increases too, leading to a high increase of the dielectric constant.
Really, the jump of the charge carriers is a thermally activated process, and therefore the dielectric constant increases with temperature.

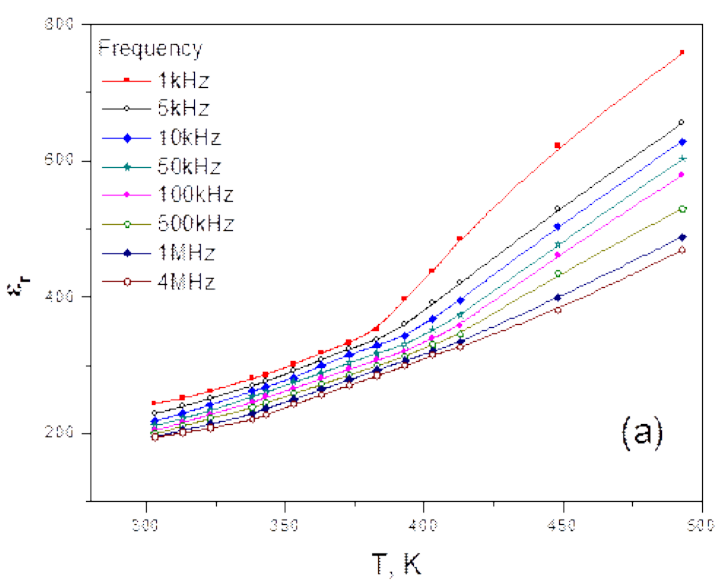

Fig. 11 Temperature dependence of the real part of the dielectric constant, $\varepsilon_{r}$, in the frequency range $1 \mathrm{kHz}-4 \mathrm{MHz}$

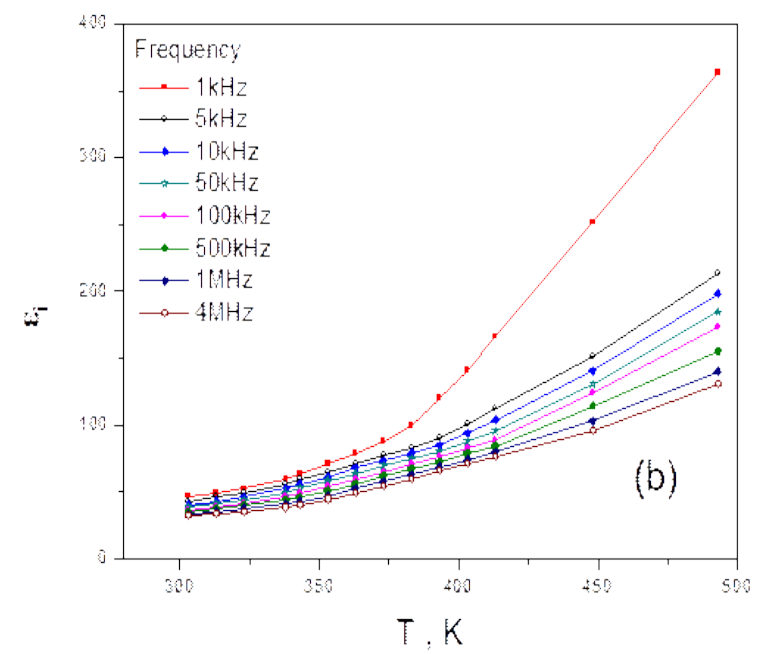

Fig. 12 Temperature dependence of the imaginary part of the dielectric constant, $\varepsilon_{i}$, in the frequency range $1 \mathrm{kHz}-4 \mathrm{MHz}$.

The dielectric loss versus frequency at various temperatures is depicted in Fig. 13. It is found that $\tan \delta$ increases with increasing temperature because of fast movement of the ions in the $\mathrm{ZnIn}_{2} \mathrm{Se}_{4}$ network and their increased response to an external electric field with increasing temperature, $\tan \delta$ increases due to an increase in conductivity of $\mathrm{ZnIn}_{2} \mathrm{Se}_{4}$ which arises from at least two different mechanisms. The first one is due to thermal activation in which the conductivity increases with increases in temperature according to the Arrhenius law. The 
second one originates from the structural changes that occur in the $\mathrm{ZnIn}_{2} \mathrm{Se}_{4}$ with temperature. During the crystallization process as shown in Fig.1, one would expect more ionic diffusion within the $\mathrm{ZnIn}_{2} \mathrm{Se}_{4}$ matrix that enhances the electrical conductivity and consequently higher $\tan \delta$. It is also observed that the $\tan \delta$ decreases nonlinearly with the increase in frequency up to a certain frequency, $f_{t}$, after which it arrives to a constant value.

The variation of $\varepsilon_{\mathrm{i}}$ with frequency is plotted as $\ln \varepsilon_{\mathrm{i}}$ versus $\ln f$ as shown in Fig. 14. Straight lines with different slopes are obtained satisfying the formula:

$$
\varepsilon_{i}=A \omega^{m}
$$

where $\mathrm{A}$ is a constant and $m$ is a parameter, calculated from the slope of $\ln \varepsilon_{\mathrm{i}}$ versus $\ln f$ curves , $m$ is related to maximum barrier height, $W_{m}$, by Guintini (Giuntini et al., 1981) relation as:

$$
m=-\frac{4 k_{B} T}{W_{m}}
$$

The variation of $W_{m}$ with temperature is depicted in the inset of Fig.14. It is shown that the value of $W_{m}$ is $0.094 \mathrm{eV}$ at $300 \mathrm{~K}$ and it decreased to $0.0096 \mathrm{eV}$ at $413 \mathrm{~K}$. It is observed that both $s$ and $W_{m}$ decreases with increasing temperature which indicates that the $\mathrm{CBH}$ is the operating conduction mechanism (Elliott. 1987and Austin et al., 1969) The decrease in $W_{m}$ with increasing temperature may be attributed to the increase of the degree of overlap of Coulomb potential wells of the considered sites.

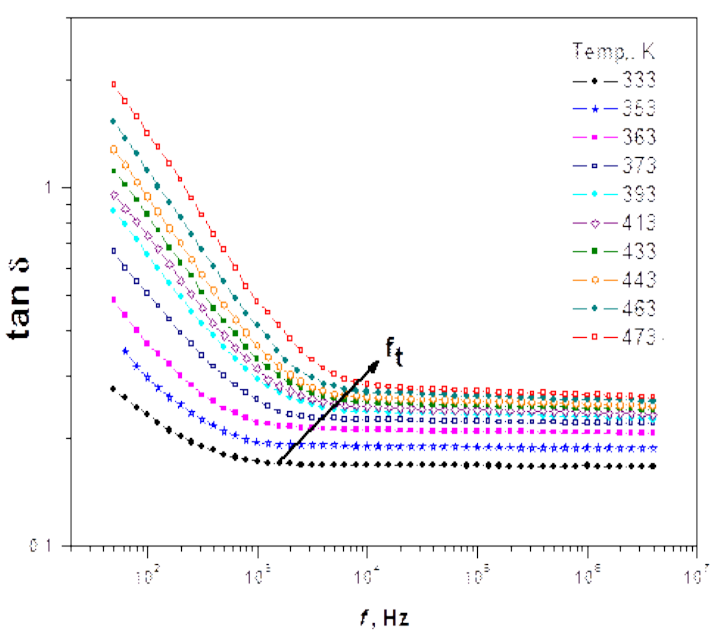

Fig. 13 Variation of dissipation factor, $\tan \delta$, with frequency, $f$, for $\mathrm{Znln}_{2} \mathrm{Se}_{4}$ thin films in the temperature range $333-473 \mathrm{~K}$.

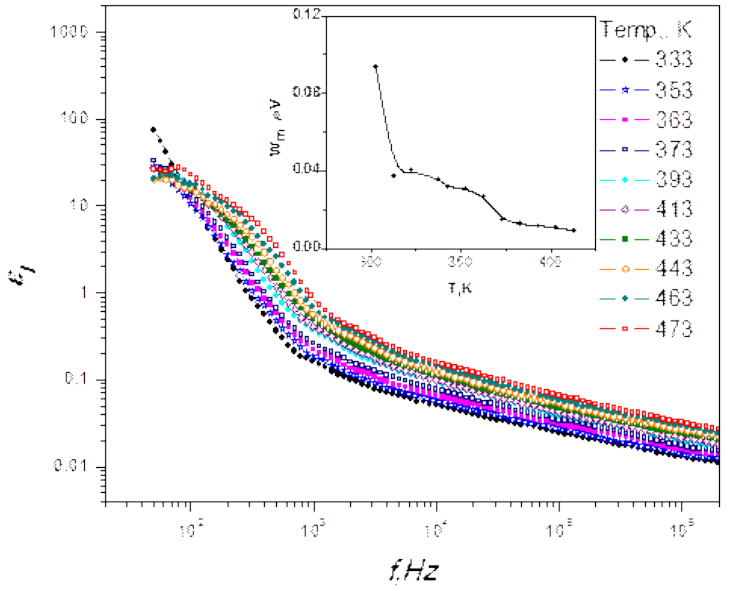

Fig. 14 Plot of $\operatorname{Ln} \varepsilon_{i}$ versus $\operatorname{Ln} f$ for $\mathrm{Znln}_{2} \mathrm{Se}_{4}$ thin films in the temperature range $333-473 \mathrm{~K}$.

\section{Conclusions}

In this work we report on structure, electrical conduction mechanisms and dielectric constants of zinc indium selenide thin films. The pristine films have nanostructure. The crystallite size of $\mathrm{ZnIn}_{2} \mathrm{Se}_{4}$ increases by annealing. Radial distribution function showed that in the first short range order shell; Se atom is tetrahedral surrounded by a vacancy and three cationic sites occupied by the metal atoms in the ratio $2 / 3$ of In and $1 / 3$ of $\mathrm{Zn}$. The medium range order region is attributed to In-In pairs having a layered structure of connected distorted octahedral. The DC conduction mechanisms depend on applied potential; namely they are consequently generation- recombination, Ohmic and exponential trap space charge limited conduction mechanisms. The activation energy is calculated and found to be $0.96 \mathrm{eV}$. The concentration of thermally generated electrons in the conduction band, $\mathrm{n}_{\mathrm{o}}$, has been estimated $4.5 \times 10^{18} \mathrm{~m}^{-3}$. The dielectric behavior of $\mathrm{ZnIn}_{2} \mathrm{Se}_{4}$ films with temperature and frequency has been discussed and the potential barrier has been determined. The decrease of potential barrier and the exponent factor with temperature confirms that correlated barrier hopping is the dominant conduction mechanism.

\section{References}

Abdel Malik TG, Abdel-Latif RM, (1997) Ohmic and space-charge limited conduction in cobalt phthalocyanine thin films Thin Solid Films 305:336-340. 
Abdullayev AG, Kerimova TG, Kyazumov MG, Khidirov AS, (1990) An electron diffraction study of the ZnIn2Se4 crystal structure: A novel phase, Thin Solid Films 1990:309-315.

Ardona MC, Christensen NE, asol GF, (1986) Terms Linear in $\mathrm{k}$ in the Band Structure of Zinc-BlendeType Semiconductors, Phys. Rev. Letter 56 : 831.

Austin L.G., Mott N.F., (1969) . Advances in Physics, Adv. Phys. $18: 41-102$.

Azim ME - Araghi, ampbel DC, rier AK, Collins RA, (1996) Electrical conduction mechanisms in thermally evaporated lead phthalocyanine thin films Semiconductor. Sci Technol., Vol . 11, pp: 39-43.

Azim-Araghi ME, (2007) Electrical properties of chloro aluminum phthalocyanine thin film sandwich devices Applied Physics 45:40-43.

Babu PReddy, MV., Revathi N, Reddy KT. Nano J. (2011). Effect of $\mathrm{Ph}$ on the physical properties of ZnIn2Se4 thin films grown by chemical bath deposition Electron. Phys.3: 25.

Choe SH., (2009) Optical energy gaps of un doped and Co-doped ZnIn2Se4 single crystals. Curr. Appl. Phys . 9:1-3.

Cullity BD., (1956) Elements of X-Ray Diffraction., Addison-Wesley, Reading, M., Dolle. H. (1979). The Influence of Multi-axial Stress States . Stress Gradients and Electric and Isotropy on the Evaluation of (residual) Stresses by X-ray , J. Appl. Crysl., 12: 489-501.

Edwards JG, Burkel p, Norwisz JP, (1999) Diffusion reaction in the $\mathrm{ZnSe} 2$ - Znln2se4 system. Thermochimic Acta 340 - 341: 323 - 339.

Elliott S.R., (1987) . A.C. conduction in amorphous chalcogenide and pintcide semiconductors. Adv. Phys. 36 : 135-217.

Elliott SR, (1978) Temperature dependence of a.c. conductivity of chalcogenide glasses Philos. Mag. B 37: 553-560.

El-Nahass MM, Attia AA, Salem GF, Ali HAM, Ismail MI, (2014), Dielectric and impedance spectral characteristics of bulk Znln2se4 Physica B 434: 89-94.

El-Nahass MM, Zeyada HM, Aziz MS, Makhlouf MM, (2005) Current transport mechanisms and photovoltaic properties of tetraphenylporphyrin /ntype silicon heterojunction solar cell Thin Solid Films 492 : 290-297.

El-Nahass MM, Zeyada HM, Aziz MS, El-Ghamaz NA, (2002), Optical properties of thermally evaporated SnS thin films. J. Mater. 20 : 159-170.

Filipowicz J, Romeo N, Tarricone L, (1981) Photoelectrical memory effect in ZnIn2Se4, Solid State Communications 38: 619 -623.

Gao L, Qian X, Zhang L, (2001) Tetratrifluoroethoxyl zinc phthalocyanine: potential photosensitizer for use in the photodynamic therapy of cancer., J. Photochem. Photobiol. 65: 35-38.
Gastaldi L. , Simeone MG, Viticoli S. (1987) Structure refinement of $\mathrm{ZnIn} 2 \mathrm{Se} 4$, Journal of Solid State Chemistry, 66:251-255.

Giuntini JC, Zanchetta JV, Jullien D, Eholie R, Houenou P, (1981) Temperature dependence of dielectric losses in chalcogenide glasses.J. NonCryst. Solids 45: 57-62.

Gould RD, (1996) Structure and electrical conduction properties of phthalocyanine thin films Coordination Chemistry Reviews 156 : 237-274.

Grilli E, Guzzi M, Molteni R. (1976) Study of the vibrational properties of InxGaAsyS quaternary alloys Phys. Stat. Sol. (a) 37:399 -406.

Hagenmuller P, (1990). Solid State Ionics. BondValence Parameters for Solids 40-41, 3 .

Hamza AA, Mabrouk MA, Ramadan WA, Emara AM, (2003) Refractive index and thickness determination of thin-films using Lloyd's interferometer. Optics Communications. 225:341 348.

Hendia TA, Soliman LI, (1995) Optical absorption behavior of evaporated $\mathrm{ZnIn} 2 \mathrm{Se} 4$ thin films, Thin Solid Films. 261:322 - 327.

Kale R B, Lokhande C D, (2005) Influence of air annealing on the structural, morphological, optical and electrical properties of chemically deposited ZnSe thin films, Appl. Surf. Sci. 252 : 929-938.

Krishnakumar KP, Menon CS, (1997) Electrical and Optical Characterization of Vacuum Evaporated Magnesium Phthalocyanine Thin Films J. Solid State Chem. 128 :27-29.

Luengo J, Joshi NV, (1996) Composition dependence of the energy gap of ZnxMn1-xIn2Se4 and optical absorption spectroscopy. Mater . Lett. 26: 47-50.

Mazen SA, Elfalaky A, Mansour SF, (1997) Electrical Conductivity of Li-Ti Ferrites Phys. Status Solidi B 201:449-456.

Mott NF, (1987) Conduction in Non Crystalline Materials, Clarendon Pres, Oxford.

Pawar S M, Pawar BS, Kim J H,(2011), Joo OS, Lokhande CD, Recent status of chemical bath deposited metal chalcogenide and metal oxide thin films, Curr. Appl.Phys. 11: 117.

Rouxel J, Moelo Y, Lafond A, Disalvo FJ, Meerschault A, Roesky R, (1994), role of Vacancies in Misfit Layered Compounds: Case of the Gadolinium Chromium Sulfide Compound, Inorg. Chem. 33, 3358-3363.

Saleh AM, Gould RD, Hassan AK, (1993) Dependence of AC electrical parameters on frequency and temperature in zinc phthalocyanine thin films Phys. Status Solidi (a) 139: 379-389.

Sedeek K, Mahmoud EA, Terra FS, El Din SM, (1994) Optical properties of amorphous chalcogenide thin films: The effect of $\mathrm{Te}$ isovalence substitution in the Ge-S-Se system. J. Phys.D Appl. Phys. 27: 156 .

Senthile K, Mangalaraj D, Narayandss SK, Hong B, Roh Y, Park CS, (2002) Argon and nitrogen 
implantation effects on the structural and optical properties of vacuum evaporated cadmium sulphide thin films. J. Yi, Semicond. Sci, Technol. 17: 97.

Shafai TS, Gould RD, (2007) Alternating current measurements of thermally evaporated triclinic lead phthalocyanine thin films. Thin solid films 516:383-387.

Soliman HS., Abdel-Hady D, Ibrahim E, (1998), Optical properties of thermally vacuum evaporated AgSbSe2 thin films. J. Phys. Condens. Matter 10: 847.

Sun X, He Y, Feng J, (2009) Growth and characterization of $\mathrm{ZnIn} 2 \mathrm{Se} 4$ buffer layer on CuInSe2 thin films, Journal of Crystal Growth 312 : $48-51$.

Vishnuvardhan TK, Kulkarni VR, Basavaraja C, Raghavendra SC, (2006) Synthesis, characterization and a.c. conductivity of polypyrrole/Y2 O3composites Bull. Mater. Sc. 29(1): 77-83.

Wiegers GA, Meetsma A, Von Smaalen S, Haange RJ, Wulf J, Zeinstra T, DeBoer TL, Kuypres S,
Tendeloo GV, Landuyt JV, Amelinekx S, Meerschaut A, Rabu R, Roxel J, (1989) Misfit layer compounds (MS)nTS2 $(\mathrm{M}=\mathrm{Sn}, \mathrm{Pb}, \mathrm{Bi}$, rare earth elements; $\mathrm{T}=\mathrm{Nb}$, $\mathrm{Ta} ; \mathrm{n}=1.08-1.19)$, a new class of layer compounds, Solid State Communications , 70 (4) : 409-413.

Yadav SP, . Shinde PS, Rajpure KY, Bhosale CH, (2008) Preparation and properties of spraydeposited ZnIn2Se4 nanocrystalline thin films, Journal of Physics and Chemistry of Solids J.Phy. Chem. 69: 1747 - 1752.

Zeyada HM, Aziz MS, Behairy AS, (2009) Absorption and dispersion studies of thermally evaporated nanocrytallite structure $\mathrm{ZnIn} 2 \mathrm{Se} 4$ thin films, Eur. Phys. J. Appl. Phys. 45:30 601.

Zeyada HM, Aziz MS, Behairy AS, (2009) Structure formation and mechanisms of DC conduction in thermally evaporated nanocrystallite structure ZnIn2Se4 thin films, Physica B 404:3957-3963.

Zeyada HM, EL -Nahass MM, Makhouf MM, (2011) Electronic transport mechanisms in tetraphenyleprophyrin thin films. Curr.Appl.Phys. 11: $1326-1331$.

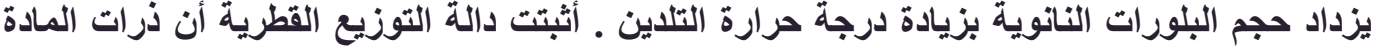

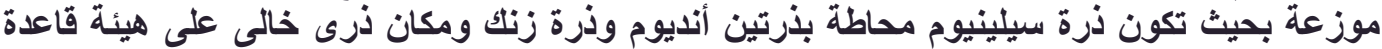

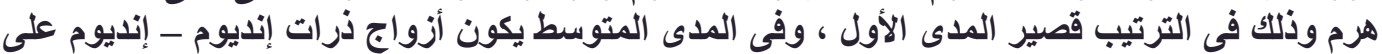

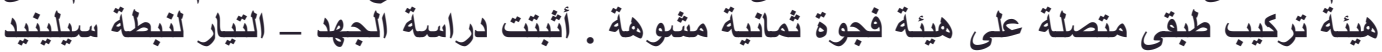

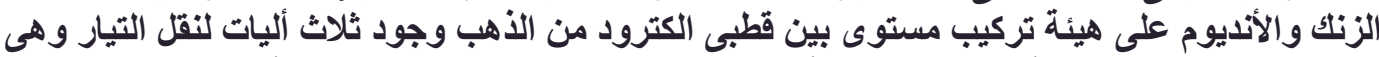

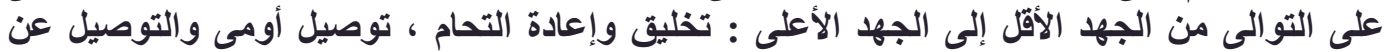

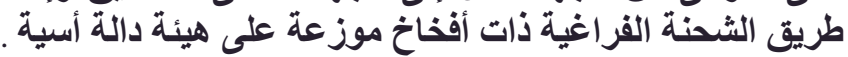

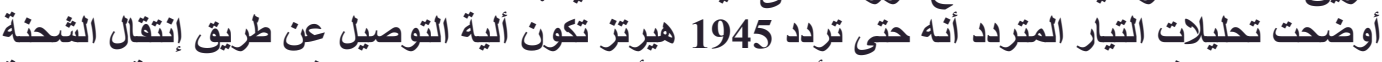

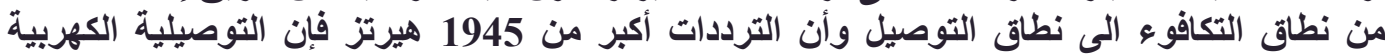

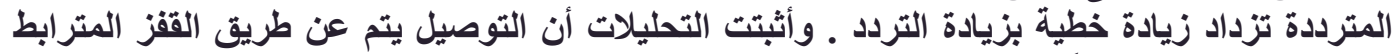

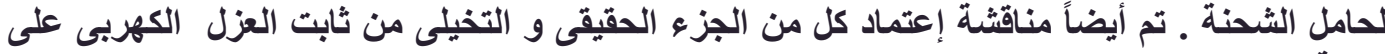

